\title{
Collective Risk Generalization to Creditrisk+
}

\author{
Reza Habibi \\ Iran Banking Institute, Central Bank of Iran, Tehran, Iran
}

Received 19-June 19, 2018

Accepted July 25, 2018

\begin{abstract}
Using the collective risk models of actuarial science, the Creditrisk+ is extended to the case of random number obligors. First, mathematical methods to compute the distribution of total loss are studied. Then, the mathematical results are applied and verified numerically. The insufficiency data in risk management is a big problem. Thus, the case of data scarce is studied using a Bayesian approach. Finally, a concluding remarks section is also given.
\end{abstract}

Keywords: Bayesian inference; Collective risk; Creditrisk+; Modelrisk; Data scarce problem; Moment generating function; Monte Carlo simulation

\section{Introduction}

In the last two decades, the credit risk modeling has been received considerable attentions in financial literatures (Avesani et al., 2006). Indeed, evaluation of the default probability of any borrower is the main concern of bankers when they lend to their clients (Liao et al. 2009). Almost for all debtors, the quantitative modeling of the credit risk is too important. To this end, some risk measures such as the credit value at risk are computed. There are two approaches for modeling credit value at risk: CreditMetrics and CreditRisk+ (Avesani et al., 2006). These approaches are applicable by regulators and risk managers which make decisions about the capital adequacy ratio. The CreditMetrics is used for rating (Lee, 2011). By this approach, the credit risk is defined as the risk that the security keepers don't materialize the security expected value because the borrower's credit quality is deteriorated (Jarrow, 2011). Using CreditRisk+ the default models are constructed. That is, in this approach, credit risk is considered as risk

that borrower of security failures on his/her promised obligations. Therefore, default of borrowers may make losses in the portfolio (Huang and $\mathrm{Yu}, 2010)$. Another difference is that the Creditrisk+ applies actuarial methodologies to derive the loss distribution of a financial portfolio. In this approach, just the default risk is modeled, and downgrade risk is not considered (Xiaohong et al., 2010)

As it is stated, the Creditrisk+ provides an actuarial based framework for quantitative credit risk management. This software computes the portfolio and other debt instruments loss distributions which lead to determination of the required economic capital. Avesani et al. (2006) reviewed basic Creditrisk+ models exist in the literatures and proposed some generalizations including latent factors and random probabilities.

Creditrisk + basic model is very similar to the individual risk model of actuarial risk theory (see, Kaas et al., 2008) at which, following notation Avesani et al. (2006), the total normalized loss $\lambda$ of $n$ obligor's normalized losses $\lambda_{i}=D_{i} v_{i}, i=1, \ldots, n$ is given by Eq. (1), as follows

$$
\lambda=\sum_{i=1}^{n} \lambda_{i}=\sum_{i=1}^{n} D_{i} v_{i}
$$

where the $i$-th default $D_{i}$ of Eq. (1) occurs (is one) with probability $p_{i}$ and is zero with probability $1-p_{i}$ and $v_{i}$ is the $i$-th normalized 
exposure. Although, Avesani et al. (2006) assumed that $v_{i}$ 's are fixed, however, it is possible to assume that they are random variables with gamma or log-normal (generally positive skewed) distributions.

A natural extension (and different of latent factors considered by Avesani et al.) to the basic model of Creditrisk+ is the collective risk model where the number of obligors $n$ who contribute exposure, itself, is also a random variable denoted by $N$. It is assumed that $N$ is independent of, $D_{i}$ 's and $v_{i}$ 's. However, usually $D_{i}$ 's and $v_{i}$ 's are correlated. In literatures, usually, selected distributions for $N$ are negative binomial and Poisson laws. Thus, like Eq. (1), the collective (credit) risk of a portfolio is presented as a random sum as

$$
\lambda=\sum_{i=1}^{N} D_{i} v_{i}
$$

When $N=0$, then $\lambda=0$. This fact implies that $\lambda$ has a mixture distribution. Thus, $\lambda$ has a compound distribution.

Although, Avesani et al. (2006) proposed an Excel addin $M C M C R+$, however, for computational purposes of some generalizations of Creditrisk+, even generalizations proposed by Avesani et al. (2006), the Modelrisk Excel add-in of Vose (2015) seems to be very useful. This paper considers the credit risk management. An interesting fact is that similar pattern of the above mentioned random sum occurs in banking operational risk management using the AMA method (see, Shevchenko, 2011).

The rest of paper is organized as follows. In the next section, mathematical results are presented and Creditrisk+ is generalized to the case of collective Creditrisk+. The numerical examples are developed in the section 3. The big problem of risk management is the scarcity of data available in hand. Hence, the data scarce case is studied using Bayesian inferential tools. Finally, a concluding remarks section is also proposed.

\section{Mathematical Expression of Creditrisk}

To compute risk measure like value at risk (VaR), the distribution of $\lambda$ is needed. As follows, some stylized facts about actuarial risk theory are updated and modified for Creditrisk+ designed for credit risk management. For each fact, necessary conditions are stated.

(a) The first fact is about the moment generating function of collective impact of credit risk. To this end, assuming all $\lambda_{i}$ 's have the same distributions, one can see that the moment generating function of $\lambda$ is given by Eq. (2), as follows

$$
M_{\lambda}(x)=M_{N}\left(\log \left(M_{\lambda_{i}}(x)\right)\right.
$$

(Kaas et al., 2008, see page 43, Eq. (3.5)). For example, when $N$ has geometric distribution with parameter $0<p<1$ and $\lambda_{i}$ 's are exponentially distributed with parameter 1 and independent, then Eq. (2) reduces to $M_{\lambda}(x)$ as follows

$$
M_{\lambda}(x)=p+(1-p) \frac{p}{p-x}
$$

which is the moment generating function of a mixture distribution. Although, there is a closed form for $M_{\lambda}(x)$, however, this is not true, generally and Monte Carlo simulation should be used.

(b) A natural method in actuarial science is the approximating the distribution of total claim. One can see that in collective risk models, the central limit theorem (CLT) approximation doesn't work well and two more accurate approximations for distribution of $\lambda$ are translated gamma (TG) and normal power (NP) approximations. Traditionally, there exist two justifications for NP approximations. First, based on approximating the distribution of $Z+\frac{\gamma_{\lambda}}{6}\left(Z^{2}-1\right)$ where $Z$ has standard normal distribution. The second justification is derived using Edgeworth expansion. To apply both TG and NP methods, the first three moments are needed. Let the cumulant generating function of $\lambda$ be 


$$
K_{\lambda}(x)=\log \left(M_{\lambda}(x)\right)
$$

Indeed, in the case of identically distributed $\lambda_{i}$ 's, then the cumulant function is given by Eq. (3), as follows

$$
K_{\lambda}(x)=K_{N}\left(K_{\lambda_{i}}(x)\right)
$$

Notice that the cumulant function of Eq. (3) is approximated by Eq. (4), as given in below

$$
\begin{aligned}
K_{\lambda}(x)= & E(\lambda) x+\operatorname{var}(\lambda) \frac{x^{2}}{2}+E\left((\lambda-\mathrm{E}(\lambda))^{3}\right) \frac{x^{3}}{6}+ \\
& O\left(x^{4}\right),
\end{aligned}
$$

(Kaas et al., 2008, see page 29).

(c) Here, the mean and variance of $\lambda$ are derived. They are given in Eq. (5)

$$
E(\lambda)=E\left(\sum_{i=1}^{N} E\left(\lambda_{i}\right)\right),
$$

$$
\operatorname{var}(\lambda)=\mathrm{E}\left(\sum_{i=1}^{N} \operatorname{var}\left(\lambda_{i}\right)\right)+\operatorname{var}\left(\sum_{i=1}^{N} E\left(\lambda_{i}\right)\right) .
$$

Again, when $\lambda_{i}$ 's are identically distributed, then these moments are reduced to the Eq. (6)

$$
\left\{\begin{array}{l}
E(\lambda)=E(N) E\left(\lambda_{i}\right), \\
\operatorname{var}(\lambda)=E(N) \operatorname{var}\left(\lambda_{i}\right)+E^{2}\left(\lambda_{i}\right) \operatorname{var}(N),
\end{array}\right.
$$

(Kaas et al., 2008, see page 29, Eqs. (3.3, 3.4)).

(d) As follows, the skew and kurtosis indices of $\lambda$ are derived. These values are necessary to find the distribution of $\lambda$. To this end, notice that the following Eq. (7) is correct that

$$
E\left((\lambda-\mathrm{E}(\lambda))^{3}\right)=E\left(\sum_{i=1}^{N} E\left(\lambda_{i}-E\left(\lambda_{i}\right)\right)^{3}\right.
$$

When $\lambda_{i}$ 's are identically distributed, then equation (7) is changed to the Eq. (8), as follows

$$
E\left((\lambda-\mathrm{E}(\lambda))^{3}\right)=E(N) E\left(\lambda_{i}-E\left(\lambda_{i}\right)\right)^{3},
$$

(Lehmann and Casella 1998, see page 29, Eq. (5.26)). Thus, to find the moments of $\lambda$, the marginal distributions of $\lambda_{i}$ 's are needed. To this end, notice that, each $\lambda_{i}$ has a mixture distribution. To see this, notice that following Eq. (9) as follows, we have

$$
\begin{aligned}
M_{\lambda_{i}}(x) & =E\left(E\left(e^{D_{i} v_{i} x} \mid D_{i}\right)\right) \\
& =E\left(M_{v_{i}}\left(D_{i} x\right)\right) \\
& =p_{i} M_{v_{i}}(x)+\left(1-p_{i}\right) .
\end{aligned}
$$

This shows that the marginal distribution of $\lambda_{i}$ is mixture of two components laws of $v_{i}$ and degenerate distribution on zero. To find moments of $\lambda_{i}$, it is seen that the following Eq. (10) is correct that

$$
\left\{\begin{array}{l}
E\left(\lambda_{i}\right)=\left.\frac{\partial M_{\lambda_{i}}(x)}{\partial x}\right|_{x=0}=p_{i} E\left(v_{i}\right), \\
E\left(\lambda_{i}^{k}\right)=p_{i} E\left(v_{i}^{k}\right), k \geq 2 .
\end{array}\right.
$$

Thus, the variance of skewness of $\lambda_{i}$ are given by Eq. (11), as follows

$$
\begin{aligned}
& \left\{\begin{array}{l}
\operatorname{var}\left(\lambda_{i}\right)=p_{i} E\left(v_{i}^{2}\right)-p_{i}^{2} E^{2}\left(v_{i}\right), \\
E\left(\left(\lambda_{i}-E\left(\lambda_{i}\right)\right)^{3}=p_{i} E\left(v_{i}^{3}\right)+2 p_{i}^{3} E^{3}\left(v_{i}\right)-\right.
\end{array}\right. \\
& 3 p_{i} E\left(v_{i}\right) E\left(v_{i}^{2}\right) \text {. }
\end{aligned}
$$

(e) Here, the density function of $\lambda$ is derived. To this end, let $f_{\lambda_{1 \rightarrow i}}^{* i}$ be the convolution of densities of $\lambda_{j}, j=$ $1, \ldots, i$ for $i \geq 1$. Then, the density function $f_{\lambda}(y)$, is given by Eq. (12) as below

$$
f_{\lambda}(y)=P(N=0)+\sum_{i=1}^{\infty} f_{\lambda_{1 \rightarrow i}}^{* i}(y) P(N=i) .
$$

Assuming $\lambda_{j}$ has normal distribution with parameter $\mu$ and variance $\sigma^{2}$, then $f_{\lambda_{1 \rightarrow i}}^{* i}(y)$ is the density of normal distribution with mean $i \mu$ and $i \sigma^{2}$. The same result is correct for $\lambda_{j}$ has Cauchy distributions, i.e. if $\lambda_{j}$ is $C(\pi, \delta)$, then $f_{\lambda_{1 \rightarrow i}}^{* i}(y)$ is the density of $C(i \pi, i \delta)$, (Kaas et al., 2008, see page 44, Eq. (3.10)). To see why the formula $f_{\lambda}(y)$ is correct, it is enough notice that the moment generating function of $\lambda$ by Eq.(3) is given by Eq. (13), as follows 


$$
\begin{aligned}
& M_{\lambda}(x)=E\left(e^{x \lambda}\right) \\
& =E\left(E\left(e^{x \lambda} \mid N\right)\right) \\
& =P(N=0)+\sum_{i=1}^{\infty} E\left(e^{x \lambda} \mid N=i\right) P(N=i) .
\end{aligned}
$$

(f) Again, the density function of $\lambda$ is derived in alternative method. Let $f_{\lambda_{1 \rightarrow i}^{* i}}(y)$ be the result of the fast Fourier transform (FFT) procedure, when $N=i$ is kept fixed and denote it by $\operatorname{FFT}_{i}(y)$, hence, it is given by Eq. (14)

$f_{\lambda}(y)=P(N=0)+\sum_{i=1}^{\infty} F F T_{i}(y) P(N=i)$.

Indeed, for each $i$, Excel add-in $M C M C R+$ gives the $F F T_{i}(y)$ and therefore, $f_{\lambda}(y)$ is obtained. Since, $P(N=i)$ decreases as $i$ gets bigger, thus, it is enough to compute $F F T_{i}(y)$ for suitable $i$ 's. The maximum $i$ 's is chosen such that $P(N \geq i)$ is negligible. The Aggregate Panjer option of Modelrisk software computes the density $f_{\lambda}(y)$ and thus the VaR is obtained.

The following proposition summarizes the above discussion.

Proposition. In a collective Creditrisk+ format, assuming $\lambda_{i}$ 's are identically distributed, then

(i) The moment generating function, mean and variance of $\lambda$ are given by Eq. (15), as follows

$$
\left\{\begin{array}{l}
M_{\lambda}(x)=M_{N}\left(\log \left(M_{\lambda_{i}}(x)\right),\right. \\
E(\lambda)=\mathrm{E}(\mathrm{N}) \mathrm{E}\left(\lambda_{i}\right) \\
\operatorname{var}(\lambda)=\mathrm{E}(\mathrm{N}) \operatorname{var}\left(\lambda_{i}\right)+E^{2}\left(\lambda_{i}\right) \operatorname{var}(N) .
\end{array}\right.
$$

(ii) Generally, $E\left(\lambda_{i}^{k}\right)=p_{i} E\left(v_{i}^{k}\right), k \geq 1$.

(iii) NP approximation. For $\lambda^{*} \geq 1$, then NP approximation is given by Eq. (16)

$$
P\left(\frac{\lambda-E(\lambda)}{\sqrt{\operatorname{var}(\lambda)}} \leq \lambda^{*}+\frac{\gamma_{\lambda}}{6}\left(\lambda^{* 2}-1\right)\right) \approx P\left(Z \leq \lambda^{*}\right) .
$$

Here, $\gamma_{\lambda}$ is the skewness of $\lambda$ and $Z$ has standard normal distribution with zero mean and variance one. (iv) $T G$ approximation. Let $F_{\lambda}$ be the distribution function of $\lambda$ and $G(., \alpha, \beta)$ be the distribution function of gamma distribution with parameters $\alpha, \beta$. Then, $F_{\lambda}\left(\lambda^{*}\right)$ is approximated as Eq. (17)

$$
F_{\lambda}\left(\lambda^{*}\right) \approx G\left(\lambda^{*}-\lambda_{0}, \alpha, \beta\right) .
$$

Here, $\lambda_{0}, \alpha, \beta$ are chosen such that $E(\lambda)=\lambda_{0}+\alpha \beta$, $\operatorname{var}(\lambda)=\alpha \beta^{2}$ and $\gamma_{\lambda}=\frac{2}{\sqrt{\alpha}}$.

(v) The density function $f_{\lambda}(y)$, is given by Eq. (18)

$$
f_{\lambda}(y)=P(N=0)+\sum_{i=1}^{\infty} F F T_{i}(y) P(N=i),
$$

for each $i$, Excel add-in $M C M C R+$ gives the $F F T_{i}(y)$.

Remark 1. Avesani et al. (2006) showed that $D_{i}$ has Poisson distribution as $p_{i}=p \rightarrow 0$. Let $v_{i}=v$. Then,

$$
\begin{gathered}
M_{\lambda}(x)=E\left(e^{x v \sum_{i=1}^{N} D_{i}}\right)=E\left(E\left(e^{x v \sum_{i=1}^{N} D_{i}} \mid N\right)\right)= \\
E\left(e^{N p\left(e^{x v}-1\right)}\right)=e^{\theta\left(e^{u}-1\right)},
\end{gathered}
$$

where $u=p\left(e^{x v}-1\right)$. Also, let $\lambda=\sum_{i=1}^{N} \lambda_{i}$ and $N$ has Poisson distribution with parameter $\theta$ and $\theta$ is a gamma variable with parameters $\alpha, \beta$, then, it is easy to see that the moment generating function is given by Eq. (19)

$$
M_{\lambda}(x)=\frac{1}{\left(1-\beta\left(e^{g(x)}-1\right)\right)^{\alpha}},
$$

where $g(x)=\log M_{\lambda_{i}}(x)$. Suppose that $\lambda_{i}$ 's have power series distributions with density given by $f_{\varphi}\left(\lambda_{i}\right)=$ $\frac{e^{\varphi \lambda_{i} h\left(\lambda_{i}\right)}}{c(\varphi)}$. The moment generating function is $M_{\lambda_{i}}(x)=$ $\frac{c(\varphi+x)}{c(\varphi)}$. It is easy to see that the moment generating function given by Eq. (20)

$$
M_{\lambda}(x)=e^{\theta\left(\frac{c(\varphi+x)}{c(\varphi)}-1\right)} .
$$

Remark 2. An alternative method to approximate distribution of $\lambda$ is to use simultaneously both Monte Carlo or bootstrap (to simulate moments of $\lambda$ ) and Edgeworth or Cornish-Fisher expansions (to simulate $F_{\lambda}$, 
i.e., the distribution function of $\lambda$ ). To this end, the Urnto-Urn method should be applied in running the Monte Carlo method to simulate mixture distributions.

\section{Examples}

Here, using some examples, the above results are applied and verified numerically.

Example 1. Let $N=1$ is observed and $v$ takes two values $a$ and $b$. Here, $\lambda$ takes three values $0, a, b$. Let $P(v=a, D=1)=p_{a} \quad$ and $\quad P(v=b, D=1)=p_{b}$ where $p_{a}+p_{b}=P(D=1)=p$. Then, $P(\lambda=a)=p_{a}$ and $P(\lambda=b)=p_{b}$. Thus, $P(v=a \mid D=1)=\frac{p_{a}}{p_{a}+p_{b}}=$ $1-P(v=b \mid D=1)$. Next, suppose that $N$ has Poisson distribution with intensity parameter 10 and $P(v=$ $a \mid D=1=0.1$ and $p=0.1$. It is seen that $p a=0.01$ and $p_{b}=0.09$. Indeed, $P(\lambda=0)=0.9, P(\lambda=a)=0.01$ and $P(\lambda=b)=0.09$. For $a=10$ and $b=20$ thousand dollars, $E\left(\lambda_{i}\right)=1.8, \quad \operatorname{var}\left(\lambda_{i}\right)=33.1$ and $E\left(\lambda_{i}-\right.$ $E(\lambda i)) 3=553.605$. Thus, $E \lambda=18, \operatorname{var} \lambda=363.4$ and $\gamma_{\lambda}=29.07$. To apply the TG approximation, it is seen that $\alpha=0.00473, \quad \beta=277.18$ and $\lambda_{0}=16.69$. However, it doesn't work well, here. Using the NP approximation, the $0.99 \mathrm{VaR}$, in this case, is 317.8 thousand dollars. The exact VaR is 240 thousand dollars.

Example 2. Suppose that obligors are categorized to three categories. The probability of each category is $p_{i}$. The amount of loss severity is $v_{i}=0, a, b$ for each category. Let $N_{1}, N_{2}, N_{3}$ be the number of obligors in the first, second and third category with intensities parameters $\theta_{1}, \theta_{2}, \theta_{3}$, respectively. Thus,

$$
\begin{gathered}
E(\lambda)=a \theta_{2} p_{2}+b \theta_{3} p_{3} \\
\operatorname{var}(\lambda)=a^{2} \operatorname{var}\left(N_{2} D_{2}\right)+b^{2} \operatorname{var}\left(N_{3} D_{3}\right)
\end{gathered}
$$

where $\operatorname{var}\left(N_{2} D_{2}\right)=\theta_{2} p_{2}+\theta_{2}^{2} p_{2}\left(1-p_{2}\right) \quad$ and $\operatorname{var}\left(N_{3} D_{3}\right)=\theta_{3} p_{3}+\theta_{3}^{2} p_{3}\left(1-p_{3}\right)$. Assuming $p_{2}=0.02, p_{3}=0.03, a=1, b=2, \theta_{2}=10 \quad$ and $\theta_{3}=20$, then the 99 percent $\mathrm{VaR}$ is .
Example 3. Assume that a portfolio contains two types of loans. The probability of default in each type is $p_{i}$. If there is a default, then the severity of default has distribution $q_{i}\left(v_{i}\right)$. Assuming each type contain $N_{i}, i=1,2$ numbers of obligors having Poisson distributions with parameters $\theta_{i}, i=1,2$.. Then,

$$
\begin{gathered}
E(\lambda)=E\left(v_{1}\right) \theta_{1} p_{1}+E\left(v_{2}\right) \theta_{2} p_{2} \\
\operatorname{var}(\lambda)=\operatorname{var}\left(N_{1} D_{1} v_{1}\right)+\operatorname{var}\left(N_{2} D_{2} v_{2}\right)
\end{gathered}
$$

Let $\theta_{1}=10, \theta_{2}=20, p_{1}=0.01, p_{2}=0.02$ and losses $v_{1}$ and $v_{2}$ are 5 or 10 dollars with probability of 0.5 and 0.5. Then, the 99 percent $\mathrm{VaR}$ is .

Example 4. Let $v_{i}=0,2$ with probability of 0.25 and 0.75 , respectively. Then the 99 percent $\mathrm{VaR}$ is. Suppose that $P\left(v_{i}=v^{*} \mid D_{i}=1\right)=q_{i}$ and $P\left(v_{i} \in\right.$ $v, v+d v=1-q i v *$ ) for $0<v<v *$. Again, let $\lambda i=v i D i$ and $N$ has Poisson distribution with intensity parameter $\theta$. Here, when $q_{i}=0.3, v^{*}=5, \theta=20$, then the 99 percent $\mathrm{VaR}$ is .

Example 5. Let $\lambda_{i}=v_{i} D_{i}$ where $v_{i}$ has exponential distribution with scale parameter $\beta$. Therefore, $\lambda_{i}=0$ with probability of $p_{i}$ and $\lambda_{i}$ has density of $\frac{1}{\beta} e^{-\frac{\lambda_{i}}{\beta}}$ with probability of $1-p_{i}$. For $p_{i}=0.05, \beta=1$, and $N$ is Poisson with parameter 5 , the 99 percent VaR is .

4 Data scare case. Data scarce is a small sample problem. Both Shevchenko (2011) and Svensson (2015) studied the operational risk management when the data is scarce. Here, their approach in operational risk managements is extended to the credit risk management. The main idea of Bayesian method to compute $f_{\lambda}$, the density of $\lambda=\sum_{i=1}^{N} \lambda_{i}$, assuming the priors for $N$ and $\lambda_{i}$ 's are given, is to derive the posterior of them and then to simulate $\lambda$, using Monte Carlo or Panjer recursion methods. The Aggregate Panjer option of Modelrisk software is good tool, in this way. Also, the Excel addin $M C M C R+$ is a useful instrument to compute the $\mathrm{VaR}$, directly. Here, throughout two examples, these methods are studied. 
Remark 3. When we take Bayesian approach to deal with it, a priori knowledge is required. How to solve this problem is worth considering. Generally, there are two approaches. First, because of computational simplicity, the conjugate priors are selected with hyper-parameters that are estimated using historical data based on empirical Bayes method. To this end, some computational methods like EM algorithms may be used (Lehmann and Casella (1998)). These approaches are chosen in the current paper. The second approach searches for suitable prior distribution for parameters to derive the posterior distributions. Some computational methods such as Monte Carlo Markov Chain (MCMC) are applied, in this case. However, in this paper, since the aim is development of Creditrisk+ and mostly data sets are simulated one, therefore, the problem of prior selection and its subjective is not the main body of study.

Example 6. Here, first, the Bayesian inference for frequency component of collective Creditrisk+ is given. Following Shevchenko and Wuthrich (2006), let $N_{1}, \ldots, N_{k}$ be a sequence of independent and identically distributed Poisson random variables with intensity parameter $\theta$, and suppose that $\theta$ has gamma distribution with parameters $\alpha, \beta$. Shevchenko and Wuthrich (2006) showed that $\theta$ has the posterior density of gamma with parameters $\hat{\alpha}_{k}$ and $\hat{\beta}_{k}$ given by $\hat{\alpha}_{k}=\alpha+\sum_{i=1}^{k} N_{i}=$ $\hat{\alpha}_{k-1}+N_{k}$ and $\hat{\beta}_{k}=\frac{\beta}{k \beta+1}=\frac{\widehat{\beta}_{k-1}}{\overline{1+\beta}_{k-1}}$ and

$$
E\left(N_{k+1} \mid N_{1}, \ldots, N_{k}\right)=z \bar{N}+(1-z) \alpha \beta,
$$

where $z$ (based on credibility theory) is the credible factor given by $z=\frac{k \beta}{k \beta+1}$. Again, the Bayesian modeling of severities are proposed. Here, in spite of Shevchenko and Wuthrich (2006) which assumes a log-normal law for severity distribution, it is assumed that $\lambda_{j}=$ $D_{j} v_{j}, j=1,2, \ldots, M$ where $D_{j}$ has Bernoulli distribution with parameter $p$ and $p$ has beta distribution with parameters $\omega, \varphi$. Thus, $p$ has beta distribution with parameters $\omega+\sum_{j=1}^{M} D_{j}$ and $\varphi+k-\sum_{j=1}^{M} D_{j}$. Also, $v_{i}$ has $\log$-normal distribution with parameters $\mu$ and $\sigma^{2}$. Here, for simplicity arguments, it is assumed that $\sigma^{2}$ is known and $\mu$ has prior normal with mean к and standard deviation $\zeta$. Again, Shevchenko and Wuthrich (2006) showed that $\mu$ has normal distribution with mean $\frac{\mathrm{M} \sigma^{2}+\zeta^{2} \sum_{j=1}^{M} v_{j}}{\mathrm{M} \sigma^{2}+\zeta^{2}}$ and variance $\frac{\sigma^{2} \zeta^{2}}{\mathrm{M} \sigma^{2}+\zeta^{2}}$. Indeed, using hyperparameters and real data, posteriors estimates of parameters are computed. Then, $D_{j}, v_{j}$, then $\lambda_{j}$ as well as $\mathrm{N}$ are simulated. Finally, using the Panjer recursive "Eq. the density of $\lambda$ are computed and the Bayesian risk measure VaR is computed

Example 7. Following Shevchenko (2006), let $\lambda=$ $\lambda^{x}+\lambda^{y}$. Here, $\lambda^{x}=\sum_{j=1}^{N_{1}} \lambda_{j}^{x}$ and $\lambda^{y}=\sum_{j=1}^{N_{2}} \lambda_{j}^{y}$ with $\lambda_{j}^{x}=D_{j}^{x} v_{j}^{x}$ and $\lambda_{j}^{y}=D_{j}^{y} v_{j}^{y}$ where $v_{j}^{x}$ and $v_{j}^{y}$ are correlated with correlation coefficient $\rho$. Indeed, Gaussian, t, Clayton, Frank and Gumbel copulas are used to model dependency between $v_{j}^{x}$ and $v_{j}^{y}$.

\section{Conclusions}

This paper considers the collective Creditrisk+ extensions to the regular Creditrisk+ version. Indeed, here, it is assumed that the number of default obligors are random obeys a specified distribution say Poisson or negative binomial. Also, the severity of exposures are assumed to be random. In the new format, some extended approaches are given to compute the density of total loss variable which is needed to calculate the $\mathrm{VaR}$ risk measure. The Bayesian solution is developed to the problem of scarcity of data as well as correlations between exposures. Extending the collective Creditrisk+ models to latent factors seems to be straightforward which is omitted.

\section{Acknowledgements}

Author thanks the referee for several improving suggestions.

\section{References}

R. G. Avesani, K. Liu, A. Mirestean and J. Salvati, Review and implementation of credit risk models in the financial sector assessment program, IMF Working Paper 06/134 (Washington: International Monetary Fund). (2006). 
Available via http:// www .imf .org /external /pubs /cat /longres.aspx ?sk=19111.

S. J. Huang, and J. Yu, Bayesian analysis of structural credit risk models with microstructure noises. Journal of Economic Dynamics \& Control 34, (2010) 2259-2272.

R. A. Jarrow, Credit market equilibrium theory and evidence: Revisiting the structural versus reduced form credit risk model debate. Finance Research Letters 8 (2011), 2-7.

R. Kaas, M. Goovaerts, J. Dhaene, and M. Denuit, Modern actuarial risk theory using R (Springer, 2006, USA).

W. C. Lee, Redefinition of the KMV model's optimal default point based on genetic-algorithms-Evidence from Taiwan. Expert Systems with Applications 38 (2011), 1010-1022.

H. H. Liao, T. K. Chen and C. W. Lu, Bank credit risk and structural credit models: Agency and information asymmetry perspectives. Journal of Banking \& Finance 33 (2009), 1520-1530.

P. V. Shevchenko, Modeling operational risk. Technical report. CSIRO division of mathematical and information sciences, Sydney (2006). Australia.

P. V. Shevchenko, Modeling operational risk using Bayesian inference (Springer, 2011, USA).

P. V. Shevchenko and M. V. Wüthrich, The structural modeling of operational risk via Bayesian inference: combining loss data with expert opinions. The Journal of Operational Risk 1 (2006), 3-26.

K. P. Svensson. A Bayesian approach to modeling operational risk when data is scarce. Master's theses in mathematical sciences. Lund University. Sweden. (2015)

D. Vose, Risk analysis. vailable:http://www.vosesoftware.com (2015).

C. Xiaohong, W. Xiaoding, and W.D Desheng, Credit risk measurement and early warning of SMEs: An empirical study of listed SMEs in China. Decision Support Systems 49 (2010), 301-310. 